Athens Journal of Health - Volume 1, Issue 3 - Pages 189-200

\title{
Paediatric Emergency Care: Improving Service Provision in the United Kingdom
}

\author{
By Carol Chamley*
}

General Practitioners and Accident and Emergency services are the two most common routes, through which children and young people are referred to specialist NHS services in the United Kingdom. In a typical year, approximately 3.6 to 4 million children attend accident and emergency departments in England, with almost half of all infants under 12 months of age, and a quarter of older children requiring this service (Kennedy 2010, Hemmingway 2011, Jones et al 2011).Situated in the West Midlands, Coventry and Warwickshire is no exception, with an estimated 30.000 children necessitating emergency care from one local NHS Trust. Despite the many challenges confronting changes to paediatric and child health services, the inertia for change provided the opportunity to improve outcomes for children and young people who require urgent or unscheduled care locally. Thus, ensuring that children and young people receive age specific care from highly qualified and skilled practitioners competent in their field of paediatric emergency care. Currently there exists a paucity of paediatric emergency nurse practitioners (PENPs) (Pritchard 2012, Feetham et al 2014) available to assess, diagnose and treat children within local emergency departments. The purpose of this service improvement is to improve quality and ensure patient safety, with a resident, skilled and knowledgeable body of paediatric emergency nurse practitioners (PENPS) across the locality. Therefore this service improvement project was supported by the Coventry and Warwickshire Workforce Locality Board, who commissioned professional bespoke education from the local University, to facilitate this ongoing service improvement, coupled with intercollegial, collaborative support and mentorship from senior nurses, clinicians and academics. Practitioners follow a bespoke educational pathway integrating theory and practice outcomes. The first PENP cohort have now completed the programme. It is proposed to follow-up with an impact study to evaluate the quality and outcomes of the service improvement, coupled with ongoing

${ }^{*}$ Senior Lecturer, Coventry University, UK.

${ }^{\dagger}$ Senior Lecturer, Coventry University, UK. 


\section{strategic planning to ensure that the compliment of PENP's remains} sustainable.

\section{Introduction: Background and Context}

Children and young people require and deserve the highest standard and quality of care. As paediatric emergency services continue to evolve it is now being recognised as a sub-speciality for training for emergency medicine and paediatrics in the United Kingdom (RCPCH 2012). During the last two decades there have been dramatic and substantial changes and challenges to the way in which urgent and unscheduled care for children and young people is provided. During the 1990s it was more likely that a family would contact their general practitioner (GP) practice if they had urgent concerns regarding their child's health, however changes to GP working practices since 2004 have resulted in the evolution of a multiplicity of emergency care settings and providers. However it is worth noting that general practitioners (GPs) and Accident \& Emergency services are the two most common routes through which children and young people are now referred to specialist National Health Service (NHS) services in the United Kingdom (UK). Children make up approximately $40 \%$ of the workload of GP practice and approximately a quarter of Emergency Department (ED) attendances are children and young people under 19 years. There has been an incremental increase in the numbers of children attending ED in England by 34.5\% (Health and Social Care Information Centre (HSCIC) 2013) and rising, and it is now estimated that in a typical year approximately 3.6- 4 million children will present to Accident and Emergency Departments in England.

The challenges associated with out-of-hours first-contact were brought into sharp focus in the UK, following the national employment contract for GPs changed in 2004, resulting in an increase in attendances to emergency departments and short admissions for children around this time (Wolfe et al 2013). Moreover in April 2011 the Department of Health replaced the single 4 hour wait time standard with eight Clinical Quality Indicators (CQIs) (Feetham et al 2014) to give a more balanced approach to care in ED departments. However in the UK, GPs receive limited postgraduate training in paediatrics but also deliver first-contact care separately from paediatricians. Whilst this model has some notable strengths, equally there co-exists evidence and reporting of suboptimum outcomes for children (Wolfe et al 2013). Unplanned re-attendance rates to ED is also used to reflect quality of care on presentation, in terms of correct diagnosis, treatment and advise (Feetham et al 2014).Development of indicators for children is also noted as challenging because of the 4Ds (Wolfe et al 2013).

- Developmental change

- Dependency

- Differential epidemiology 
- Demographics

Health services for children are an important modifiable determinant of health however European health systems have been slower to adapt to the changing patterns of childhood mortality and morbidity. Furthermore, concerns for the quality of care in the UK were raised because doctors from EU countries have been recruited to provide out-of-hours primary care, a situation which raised some fundamental concerns relating to EU regulations and the free movement of professionals between countries despite differences in medical training particularly with children (Wolfe at al 2013).

One of the major challenges facing professionals is how to distinguish between serious and potentially life threatening episodes, from minor problems? Children present very differently to adults particularly experiencing different and unique exposures and conditions which increase their vulnerability. This coupled with dynamic developmental anatomy and physiology which may influence the presentation of disease/illness during childhood and potentially there can be blurring between 'minor' and 'major' as the child's condition escalates. Thus one of the greatest challenges facing health care professionals working with children is distinguishing potentially serious life threatening illness from minor problems. It is however estimated that between $17-57 \%$ of children and young people attending emergency departments, have problems judged by clinicians to be non-urgent/ minor problems and could be dealt with, with in primary care settings (Wolfe et al 2013).

The pathways for urgent, non scheduled care are complex and increasingly evidence suggests that parents seek medical advice from multiple sources such as:

GP, Pharmacist /Chemist or Out -of -Hours Services. Moreover parents are presenting with their children to Emergency Departments which is seen as a default position with the provision of 24 hour NHS service coupled with the desire for health problems to be addressed quickly by qualified practitioners (Kennedy 2012) However Feetham et al (2014) alert us to the complexities associated with families seeking medical assistance for their children, which may include such issues as parental anxiety, quality of 'safety netting', advice given during first contact and/or lack of paediatric-focused community services.

Coventry and Warwickshire is no exception with an estimated 30,000 children necessitating emergency care from the local NHS Trust (2011),increasing to $\mathbf{3 0 , 8 2 8}$ during 2012-2013.Despite these many challenges this provided the opportunity to improve outcomes for children and young people requiring urgent unscheduled care across the locality. Coventry is located in the West Midlands and is recognized as England's 9th largest city, with a population of approximately 300,000 of which 72,000 are children (approx), and $25 \%$ of the population are under 20 years of age. A mixed health 
economy is offered by such services as GP and out of hours services, NHS and private health care, coupled with Pharmacy services and over the counter products.

\section{An Established Service: Children's Emergency Department (CED) University Hospital Coventry \& Warwickshire (UHCW) United Kingdom}

Historically there were no established paediatric emergency nurse practitioners (PENPs) (Pritchard 2012, Feetham et al 2014), assessing/ diagnosing/treating children within CED. During 2007, the Privately Financed Initiative (PFI) hospital development was inclusive of a dedicated Children's Emergency Department (CED), albeit adult nurses were successfully working as emergency practitioners within the adult emergency department, who were usually designated Band 6 as evident at other emergency units. There is an urgent move to improve paediatric emergency care in light of changing need, demographics, cultural perspectives, epidemiology, social and healthcare scaffolding of childhood, policy drivers emerging technologies and advances in medical science. Moreover the role of emergency nurse practitioners role has been scrutinized in the literature (Sakr et al 2002,2003; RCN 2004; RCPCH 2011,NMCAC 2013 ) with emerging evidence substantiating the need for Paediatric Emergency Care Practitioners (PENPs) (Pritchard 2012, Feetham 2014). Nurse practitioners have been noted as more proficient than junior doctors at recording medical history and there are fewer unplanned follow -up attendances regarding patients seeking advice (Feetham et al 2014). Therefore the potential impact of adequately trained and well supported PENP's to deliver emergency unscheduled care and management to children and young people and associated workload management would be immense. There are however recognized limitations and exclusions to their role which are related to complex injuries/conditions (Table 1), but those limitations will also be attributable to junior doctors. In reality the developed skills and knowledge base of a retained speciality may lead them to be more experienced in either specific paediatric illness or injury.

Table 1. Conservative 'Exclusions' (will not/may not Meet Inclusion Criteria)

\begin{tabular}{|l|}
\hline Cardiac conditions \\
\hline Central nervous system (not including strokes) \\
\hline Child protection \\
\hline Dental/traumatic loss of teeth \\
\hline Dislocations/amputation/Joint injury \\
\hline Nerve Injuries \\
\hline Diabetes \\
\hline Max Fax \\
\hline Moderate/Severe Head Injury \\
\hline Near Drowning \\
\hline Psychological conditions \\
\hline Haemophilia and other coagulopathy \\
\hline Miscellaneous others \\
\hline
\end{tabular}


Issues of concern which were raised with commissioners during 2013 (RCGP) (2013) included:

- Increase in unscheduled attendances (Primary care)

- Inappropriate A and E attendances

- Neonatal feeding problems

- Prenatal and adolescent mental health issues

- School issues-exclusion of very young children with troublesome behaviour

- Children with chronic and long-term illness

- Transition Issues

- Safeguarding and looked after children

Therefore it further suggested that resident skilled Paediatric Emergency Nurse Practitioners have a professional knowledge base which is specifically applied to child development, developmental anatomy and physiology, specific childhood disease and treatment protocols, prescribing for children, future focussed transitional care and safeguarding than the experience or knowledge base, of junior emergency department doctors who are transient -not resident. It also seems very likely that in general, ENP services will become more widespread throughout the UK and this would reflect the international model which is offered in countries such as Canada and the USA.

Whilst it was recognized that there were incremental increases in the numbers of children attending ED in England by 34.5\% (HSCIC 2013) and rising, it was further acknowledged that health care professionals need to be adequately prepared to assess and observe children and young people, and have the ability to make appropriate clinical decisions regarding the most suitable treatment process and location. Furthermore presentations (minors) to CED would suggest strongly that it is not only possible to direct CPD education, but ultimately this will guide practitioner's scope of practice (Nursing and Midwifery Council (NMC) 2012) and subsequently a prescribing focus for PENPs. In essence, the figures captured 14,829 from 16,237 presentations in 21 sub-categories (Table 2) which in turn account for an estimated 50\% $(49.3 \%)$ of all presentations to the emergency department. These presentations account for $61 \%$ of all discharges from the emergency department. 
Table 2. Presentations to CED (Minors)

\begin{tabular}{|c|c|}
\hline $\begin{array}{c}\text { Figure 2.21 Collated Diagnoses } \\
\text { (Discharged within 4 hours).Coding } \\
\text { Categories }\end{array}$ & Numbers of paediatric presentations \\
\hline Soft tissue injury/sprains/fractures & 1765 \\
\hline Respiratory (VIW, asthma, URTI) & 1746 \\
\hline Blank (no data available) & 1616 \\
\hline Minor HI + Headache & 1428 \\
\hline Laceration & 1268 \\
\hline Diagnosis not classifiable & 1074 \\
\hline Non-specific viral illness & 921 \\
\hline Diarrhoea/gastroenteritis & 914 \\
\hline ENT/Tonsillitis/ Epistaxis & 823 \\
\hline Nothing abnormal/normal or & 624 \\
\hline Crying baby/Parents concerned & 509 \\
\hline Abrasions/contusions & 492 \\
\hline Dermatology/Eczema/Rash & 291 \\
\hline Burns/Bites/Stings/Scalds & 280 \\
\hline Foreign Body & 211 \\
\hline Eye Conditions/conjunctivitis & 183 \\
\hline Allergy and local allergic reaction & 180 \\
\hline Seizures/febrile convulsions & 141 \\
\hline Urology/UTI and Cystitis & 133 \\
\hline Poisoning (O/D) & 103 \\
\hline Abdominal Pain & 87 \\
\hline Constipation & \\
\hline &
\end{tabular}

Importantly these presentations evidenced the drivers for change, and potential for the development of a bespoke programme of learning, to develop Paediatric Emergency Nurse Practitioners (PENPs) (Pritchard 2012 Feetham 2014). Furthermore, Kennedy (2012) states that at the heart of any system providing services for children and young people are the professionals. The NHS intends to respond to the needs of children, young people and families rather than expecting them to respond to the system.

\section{Vision and Project Aims}

Within the acute sector, services were being remodelled and reconfigured whilst considering the implications for service delivery. Funding was made available from the local 'Workforce Planning Board' to establish autonomous Paediatric Emergency Nurse Practitioners in collaboration with Coventry University with due consideration given to the best available model of education and practice. The Care Quality Commissions 'Essential Standards of Quality and Safety (CQC 2010) state that providers should ensure that staff:

- Have the relevant qualifications, knowledge, skills and experience to carry out their role (CQC Outcome 12B). 
- Acquire further skills and qualifications that are relevant to the work they undertake. (CQC Outcome 14)

- Ensure children who use services can be confident that they are treated by staff who are appropriately trained to provide care, treatment and support for children. (CQC Outcome 14B).

The initiative had to embrace meeting governance whilst implementing clinical quality indicators $(\mathrm{CEM} / \mathrm{DOH})$, together with 'Time to Treatment' standards with a median time to treatment of less than 60 minutes, and being seen by a clinician (a suitably experienced Doctor or Emergency Nurse Practitioner). Also avoiding the 'Hello clinician' and excessive use of junior medical staff, who may lack experience to make definitive clinical decisions (CEM 2011).By enhancing the care delivered to this population of children and young people, the Paediatric Emergency Care Practitioner (PENP) (Pritchard 2012, Feetham et al 2014) role embraces caring for children and young people in emergency care settings, and to this end practitioners require a specific and robust skills set and knowledge base, to deliver urgent unscheduled care. Thus specific training and competency development is essential if children's emergency treatment and management is to be delivered safely, competently and to the highest possible standards. PENPs therefore deliver age specific care, delivered by qualified RN child practitioners who are educated and skilled in the assessment, diagnosis and management of children and young people thus benefitting the lives of children young people and their families, as they are seen (triaged) diagnosed and treated quicker .By engaging in specific modules delivered by Coventry University Faculty of Health and Life Sciences, a core body of practitioners have now been up-skilled in the assessment, diagnosis and management of children and young people, presenting to accident and emergency departments across Coventry and Warwickshire. As a Higher Education provider Coventry University www. coventry.ac.uk strives to achieve excellence in academic, professional, research and creative practice standards. The University aims to attract students from the national and international learning communities, aspiring to be a dynamic, global, enterprising university committed to engaging its students in a community of learning and practice. There is a strong profile for on-line learning and a dedicated research centre for children and families (CCFAR) www.coventry.ac.uk/ccfar

\section{Project Aims}

- Develop Extended Scope Practitioners (Paediatric Emergency Nurse Practitioners) to assess, diagnose, treat and discharge/refer children with minor injuries/illness

- Improve quality and ensure safety with a resident, skilled and educated body of practitioners. 
- Utilise the advanced skills/knowledge of the medical personnel within the three hospital settings

- Ensure timely access to treatment deploying ESP at highlighted increased demand times

- Provide senior medical staff with allocation of complex/seriously ill children as department continues to operate effectively

\section{Bespoke Programme of Learning}

A collaborative approach between the local Acute Trust and the HEI worked towards developing a bespoke programme of learning with competency led practice, which involved student practitioners undertaking four modules of learning which included:

- Advanced Health Assessment

- Non medical nurse prescribing (1 \& 2)

- CPD Minor Injuries

This was integrated with clinical mentorship, academic support and competency led practice.

Student Practitioners State that......

'I feel that my assessment skills have greatly improved and I feel confident to assess, treat, plan care and discharge patients.

I am currently prescribing for children I assess and provide analgesia quickly without waiting for a doctor'.

I feel it is a very valuable course and the PENP role will greatly improve the care that the patients are receiving.

By being able to 'see and treat' minor injuries and illness the children are assessed and treated in a timely manner'.

Practitioners have now completed their programme $(n=8)(2$ non completers) and primary data demonstrates successful role adoption and autonomy in line with numbers of clinical presentations to the department steadily rising year after year and a significant proportion of those presentations (> 50\%)(conservative estimate) are eligible to be seen by PENP for the entire care episode. Whilst there is limited evidence in the literature reflecting the role of the PENP, one service evaluation demonstrates a high level of patient satisfaction in the PENP management of the child's healthcare journey with minor injury and illness (Feetham et al 2014). Therefore further research is much needed to evaluate the role and substantiate the need for such crucial services which will potentially contribute to better health outcomes and survival rates for children and young people. Child survival has improved greatly in the past three decades in 15 European countries reflected by improvements in public health coupled with wider societal factors. Wolfe et al 
(2013) state that there is however wide variation in child health outcomes between these countries, as well as within each country moreover these variations are situated within health outcomes, life chances and mortality. It was found that in terms of health outcomes the United Kingdom does not compare well with its European counterparts for example the UK has the highest rates of Asthma deaths among children aged 6-7 and 13-14 (Wolfe et al 2013, Wang et al 2014).

Table 3. Global, Regional and National Levels of Neonatal, Infant and under 5s Mortality during 1990-2013. Top: 20 Western European Countries by Death per 1,000 live Births for Under 5 's (Wang et al 2014)

\begin{tabular}{|c|c|c|}
\hline Ranking & Country & $\begin{array}{c}\text { Deaths per } 1,000 \text { live } \\
\text { births for under 5s }\end{array}$ \\
\hline & & 2.4 \\
\hline 1. & Iceland & 2.6 \\
\hline 2. & Andorra & 2.7 \\
\hline 3. & Sweden & 2.8 \\
\hline 4. & Luxembourg & 3.0 \\
\hline 5. & Finland & 3.0 \\
\hline 6. & Norway & 3.5 \\
\hline 7. & Portugal & 3.6 \\
\hline 8. & Germany & 3.6 \\
\hline 9. & Spain & 3.7 \\
\hline 10. & France & 3.7 \\
\hline 11. & Italy & 3.8 \\
\hline 12. & Denmark & 4.0 \\
\hline 13. & Greece & 4.1 \\
\hline 14. & Austria & 4.1 \\
\hline 15. & Cyprus & 4.2 \\
\hline 16. & Netherlands & 4.2 \\
\hline 17. & Belgium & 4.3 \\
\hline 18. & Israel & 4.3 \\
\hline 19. & Switzerland & 4.9 \\
\hline 20. & UNITED KINGDOM & \\
\hline
\end{tabular}

\section{Closing Thoughts: The Way Forward}

Wolfe et al (2013) allude to children's services often being an afterthought when health information systems are developed. They state that as climate change experts study future scenarios for global warming, so should child health experts develop modelling techniques to examine the future of our children's health thus enabling far-sighted policy development embracing a rights -based approach to child health services (UN Convention on the Rights of the Child 1989). However much more needs to be done to translate high level goals into policy at national and international level. Therefore as this 
project has evolved it has become apparent that the way forward would be to propose:

- An evaluative study to evaluate the quality and outcomes of service improvement

- Develop a dedicated 'Tool Kit' and Competencies

- Disseminate work internationally

Finally, although the NHS has responsibilities for healthcare across the life span in reality it has been largely developed and designed by adults for adults and has been persistently been informed by the interests of adults rather than those of children and young people. Adopting a child -friendly health care approach which looks through a 'child friendly lens' will potentially maximize opportunities to improve health outcomes for children and young people by cocreating alignment and synergy between the various stakeholders, which must include children, young people and families (DH 2013).

\section{References}

Care Quality Commission (2010) Essential Standards of Quality and Safety. London. CQC.

Coventry Children and Young People's Plan 2011-2014 (2011) CYPSP available from www.coventrycypsp.org.uk Accessed 12/4/14.

Department of Health (2013) Report of the Children and Young People's Health Outcomes Forum. London. DH.

Feetham,J.Christian,W.Benger,J.Hoskins,R.Odd,D.Lyttle,D.(2014) Paediatric ED Attendance Rates: Comparing Nurse Practitioner and Other Clinicians. British Medical Journal. www.emj.bmj.com

Health and Social Care Information Centre (2013)(HSCIC) www.hscic.gov.uk

Hemmingway,P.(2011) Children and Young People's Participation in Healthcare Consultations in the Emergency Department. International Emergency Nursing 19 (4) : 192-198.

http://cmis.coventry.gov.uk.

http://www.cqc.org.uk/sites/default/files/documents/gac_-_dec_2011_update.pdf

Jones,S.Atenstaedt,R.Charles,J.Tinker,A.Richardson,G.Jones,A.(2011).Paediatric and Child Health Services literature Review. Available on line at www.2nphs. wales.nhs.uk:8080.../... Accessed 10/3/2014

Kennedy, I. (2010) Getting it Right for Children and Young People: Overcoming Cultural Barriers in the NHS to Meet Their Needs. London. DH

Nursing and Midwifery Council (2012) Role Boundaries. London. NMC.

Nursing and Midwifery Advisory Committee (2013) Extended Scope of Practice Policy for Registered Nurses and Midwives (2013).London. NMC. www.nmc. ork.uk

Pritchard, J. (2012) Overview: Extended Scope Practitioners Report. Paediatric Emergency Care Nurse Practitioners. UHCW Internal Report (With Permission).

Royal College of Nursing (2004) Services for Children and Young People: Preparing for Future Roles. London.RCN www.rcn.org.uk 
Royal College of Paediatrics and Child Health (2011) Facing the Future: Standards for Paediatric Services. London. RCPCH.

Royal College of Paediatrics and Child Health (RCPCH) (2012) Standards for Children and Young People in Emergency Care Settings. London. RCPCH. www.rcpch.ac.uk/emergencycare

Royal College of General Practitioners (RCGP) (2013) Commissioning A Good Child Health Service. London. RCGP

The Royal College of Emergency Medicine (2011) Emergency Department Clinical Quality Indicators: A CEM Guide to Implementation. London. CEM.

Sakr, M. Kendal, R. Angus, J. Sanders, A. Nicholl, J. Wardrope, J. (2002)Systematic Review of Whether Nurse Practitioners Working in Primary Care Can Provide Equivalent to Doctors? British Medical Journal: 819-823.

Sakr, M. Kendal, R. Angus, J. Sanders, A. Nicholl, J. Wardrope, J.(2003)Emergency Nurse Practitioners: A Three part Study in Clinical and Cost Effectiveness. Emergency Medicine Journal. 20 (2):158-163.

Silver, H. Ford, L. Day, L.(1968) The Pediatric Nurse-Practitioner Programme: Expanding the Role of the Nurse to Provide Increased Health Care for Children. Denver. University of Colarado.

Wang, H. et al (2014) Global, Regional and National levels of Neonatal, Infant, and

Under-5 Mortality During 1990-2013: A Systematic Analysis for the Global Burden of Disease Study 2013. London. The Lancet. www.lancet.com

Wolfe, I, Thompson, M. Gill, P. Tamburlini, G. Blair, M.Van den Bruel, A. Ehrich, J. Pettoello-Mantovani,M.Janson, Karanikolos, S. McKee,M.(2013) Health Services for Children in Western Europe www.lancet.com 
